PREPARED FOR THE U.S. DEPARTMENT OF ENERGY, UNDER CONTRACT DE-AC02-76CH03073

PPPL-3770

PPPL-3770

UC-70

Simulation Studies of the Role of Reconnection

in the "Current Hole" Experiments in the Joint European Torus

by

J.A. Breslau, S.C. Jardin and W. Park

January 2003

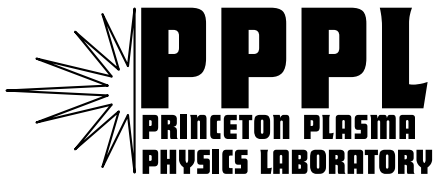

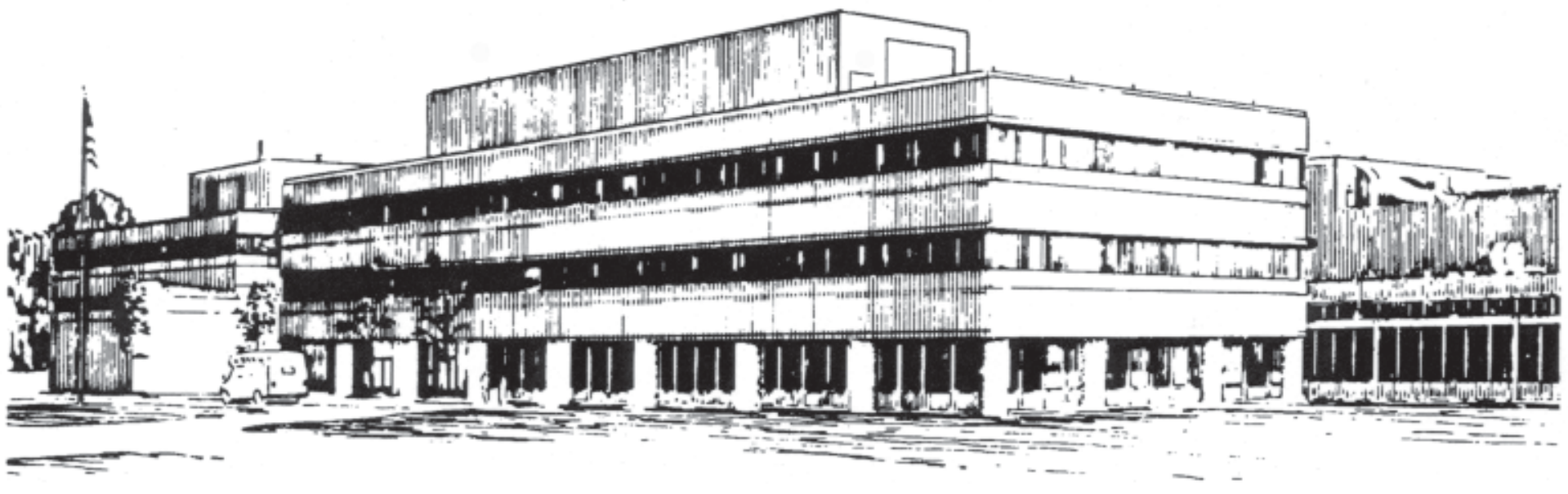

PRINCETON PLASMA PHYSICS LABORATORY PRINCETON UNIVERSITY, PRINCETON, NEW JERSEY 


\section{PPPL Reports Disclaimer}

This report was prepared as an account of work sponsored by an agency of the United States Government. Neither the United States Government nor any agency thereof, nor any of their employees, makes any warranty, express or implied, or assumes any legal liability or responsibility for the accuracy, completeness, or usefulness of any information, apparatus, product, or process disclosed, or represents that its use would not infringe privately owned rights. Reference herein to any specific commercial product, process, or service by trade name, trademark, manufacturer, or otherwise, does not necessarily constitute or imply its endorsement, recommendation, or favoring by the United States Government or any agency thereof. The views and opinions of authors expressed herein do not necessarily state or reflect those of the United States Government or any agency thereof.

\section{Availability}

This report is posted on the U.S. Department of Energy's Princeton Plasma Physics Laboratory Publications and Reports web site in Fiscal Year 2003. The home page for PPPL Reports and Publications is: http://www.pppl.gov/pub_report/

DOE and DOE Contractors can obtain copies of this report from:

U.S. Department of Energy

Office of Scientific and Technical Information

DOE Technical Information Services (DTIS)

P.O. Box 62

Oak Ridge, TN 37831

Telephone: (865) 576-8401

Fax: (865) 576-5728

Email: reports@adonis.osti.gov

This report is available to the general public from:

National Technical Information Service

U.S. Department of Commerce

5285 Port Royal Road

Springfield, VA 22161

Telephone: $1-800-553-6847$ or

(703) $605-6000$

Fax: (703) 321-8547

Internet: http://www.ntis.gov/ordering.htm 


\title{
Simulation studies of the role of reconnection in the "current hole" experiments in the Joint European Torus
}

\author{
J.A. Breslau, S.C. Jardin, W. Park \\ Princeton Plasma Physics Laboratory, P.O. Box 451, Princeton, New Jersey 08543
}

\begin{abstract}
Injection of lower hybrid current drive into the current ramp-up phase of Joint European Torus (JET) discharges has been observed to produce an annular current distribution with a core region of essentially zero current density [Hawkes, et al., Phys. Rev. Lett. 87, 115001 (2001)]. Similar "current holes" have been observed in Japan Atomic Energy Research Institute (JAERI) Tokamak 60 Upgrade (JT-60U) discharges with off-axis current drive supplied by bootstrap current [T. Fujita, et al., Phys. Rev. Lett. 87, 245001 (2001)]. In both cases, the central current does not go negative although current diffusion calculations indicate that there is sufficient non-inductive current drive for this to occur. This is explained by Multi-level 3D code (M3D) nonlinear 2D and 3D resistive magnetohydrodynamic (MHD) simulations in toroidal geometry, which predict that these discharges undergo $n=0$ reconnection events - "axisymmetric sawteeth" - that redistribute the current to hold its core density near zero. Unlike conventional sawteeth, these events retain the symmetry of the equilibrium, and thus are best viewed as a transient loss of equilibrium caused when an $\iota=0$ rational surface enters the plasma. If the current density profile has a central minimum, this surface will enter on axis; otherwise it will enter off-axis. In the first case, the reconnection is limited to a small region around the axis and clamps the core current at zero. In the second case, more typical of the JET experiments, the core current takes on a finite negative value before the $\iota=0$ surface appears, resulting in discrete periodic axisymmetric sawtooth events with a finite minor radius. Interpretation of the simulation results is given in terms of analytic equilibrium theory, and the relation to conventional sawteeth and to a recent reduced-MHD analysis of this phenomenon in cylindrical geometry [Huysmans, et al., Phys. Rev. Lett. 87, 245002 (2001)] is discussed.
\end{abstract}

\section{Introduction}

A great deal of interest has recently been generated by reports from experimental teams at the Joint European Torus (JET) ${ }^{1}$ and JAERI Tokamak 60 (JT-60) ${ }^{2}$ tokamaks of a newly observed phenomenon in which, following the application of large off-axis toroidal current drive sources, a region develops in the plasma around the nominal magnetic axis in which the toroidal current density is zero to within the measurement error. A noteworthy feature of this "current hole" region is that it defies ${ }^{3}$ the prediction made by a simple one-dimensional numerical model: that the transient effect of strong off-axis current drive will be the induction of negative current in the center, which should persist until the driven current has time to diffuse inward resistively. Still more remarkable is the fact that, despite the absence of the current that would be needed to give rise to a finite rotational transform in the core, and thus, presumably, of regular nested flux surfaces in that region, these equilibria show good confinement ${ }^{4}$ and are quite stable, with the current hole remaining intact for the entire several-second duration of the discharge. Clearly, any satisfactory explanation of this phenomenon must account for both these unusual effects.

The first problem to be confronted, however, regards the mere existence of current hole equilibria. In Greene, Johnson, and Weimer's classic 1971 paper on tokamak equilibrium, ${ }^{5}$ an expression was derived for the Shafranov shift which appears to diverge in regions of zero toroiodal current, suggesting that configurations containing such regions are unphysical. 
A recent re-evaluation of that analysis by Chu and Parks, however, has shown that this is not the case. ${ }^{6}$ By matching zero-current and finite-current solutions to the first few orders in GJW's large-aspect-ratio expansion of the equilibrium equations, they have shown that there are physical current hole equilibria that are expected to be accessible (and, additionally, negative current equilibria that are not). The question of whether the JET and JT-60 current hole plasmas are actually in this sort of equilibrium, and, if so, how they arrive at it, remains to be decided.

In this paper, we employ the parallel 3D nonlinear extended magnetohydrodynamics

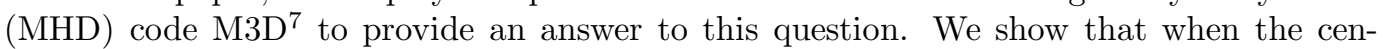
tral current density is reduced inductively below a threshold negative value, a surface with zero rotational transform $\iota$ (or safety factor $q=\infty$ ) appears in the plasma, either at the magnetic axis or at finite minor radius. This leads immediately to an axisymmetric motion in which the core region interior to the $\iota=0$ surface shifts outward, undergoing a sawtooth-like reconnection. The effect of this reconnection is to eliminate the negative current, effectively clamping its value near zero. Under certain circumstances, such "axisymmetric sawteeth" are expected to repeat for many cycles. The effect is robust enough to persist in three-dimensional MHD modeling, confirming the predictions of previous 1D reduced MHD cylindrical ${ }^{8}$ and toroidal ${ }^{9}$ and $2 \mathrm{D}$ full $\mathrm{MHD}^{10,11}$ studies.

\section{Predictions of the 1D Model}

Our reference experiment is discharge number 53488 in JET. ${ }^{1}$ Motional Stark effect (MSE) measurments taken $3.1 \mathrm{~s}$ into this shot, following about $1.6 \mathrm{~s}$ of off-axis lower hybrid current drive, indicate that the core toroidal current density is essentially zero out to approximately one-third of the plasma minor radius. The electron temperature profile has a high plateau across the current hole region at this instant and the density profile is flat. The discharge continues for several more seconds following the MSE measurement without substantial energy loss from the core.

The standard way in which to attempt to numerically model such an experiment is with a 2D equilibrium/transport code, such as the Tokamak Simulation Code (TSC), ${ }^{12}$ which is designed to predict the gross behavior of tokamak plasmas on the transport timescale by evolving through a sequence of two-dimensional MHD equilibria. Because this code employs flux coordinates, however, it breaks down when the $\iota=0$ surface enters the plasma; at that point, the surfaces traced out by the magnetic field lines are no longer uniquely labeled by their enclosed poloidal flux. To follow the evolution of the current density beyond the breakdown point, a 1D code using a cylindrically symmetric equilibrium, and the minor radius $r$ as the space coordinate, was applied to this problem. This code, informally titled "GOJET", simply evolves the 1D magnetic field diffusion equation with a source term,

$$
\frac{\partial \psi}{\partial t}=\eta\left[\frac{1}{r} \frac{\partial}{\partial r}\left(r \frac{\partial \psi}{\partial r}\right)-J_{C D}\right],
$$

where $\psi$ is the poloidal flux, $\eta$ is a constant resistivity, and $J_{C D}$ is a non-evolving analytic fit to the plasma current density response to the lower hybrid current drive as predicted by ray tracing calculations performed by the Lower hybrid Simulation Code (LSC). ${ }^{13}$ The response of the current profile to the sudden application of an off-axis source is shown in Fig. 1. Is is seen that in the absence of any $2 \mathrm{D}$ reconnection events, a large negative value will develop on axis in stark disagreement with the experimental results. It is therefore clear that more sophisticated modeling is required. 


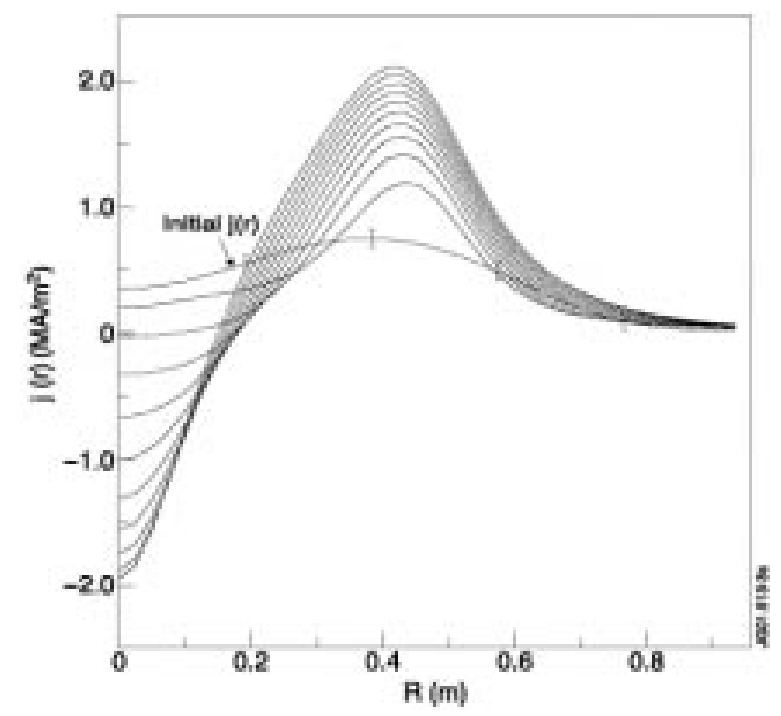

Figure 1: The current density evolution predicted by the 1D tokamak transport code.

\section{The 2D Current Hole Problem}

We next turn to full two-dimensional modeling of the discharge with the M3D code, which takes MHD equilibria as input and evolves them nonlinearly. In order to model off-axis current drive numerically, we modify the usual MHD Ohm's law equation to read

$$
\mathbf{E}+\mathbf{v} \times \mathbf{B}=\eta\left(\mathbf{J}-\mathbf{J}_{\mathbf{C D}}\right)
$$

where the drive term $\mathbf{J}_{\mathbf{C D}}=J_{C D} R_{0} \nabla \varphi$ is given by the analytic expression

$$
J_{C D}(r)=c_{1}+c_{2} r^{2}\left[\frac{1-c_{3} r^{2}}{c_{4}^{2}+\left(r-c_{5}\right)^{2}}\right] .
$$

Here $\varphi$ is the symmetry angle, $R_{0}$ is the nominal major radius, $\eta$ is the plasma resistivity (assumed constant) and $r$ is the normalized minor radius. For all further cases discussed in this paper, the parameters chosen are $c_{1}=9.2092 \times 10^{-2} ; c_{2}=0.1003 ; c_{3} \approx 1.214924$; $c_{4}=0.15$; and $c_{5}=0.54$, producing the hollow current drive profile shown in Fig. 2 . The profile is peaked at about $r=0.56$, is small but finite on axis, and falls to zero at the plasma edge. This form gave a reasonable fit to the results of ray-tracing calculations using LSC.

\section{A. JET cross-section}

We start with a $2 \mathrm{D}$ reference numerical JET equilibrium with $\beta=2 \mu_{0}\langle p\rangle / B_{T}^{2} \approx 9 \times 10^{-4}$. The current drive term is switched on at time $t=0$, and the plasma state is evolved in $2 \mathrm{D}$ (assuming axisymmetry) using the nonlinear, single-fluid MHD version of the M3D code.

The initial response of the plasma in the simulation is essentially the same as that predicted by the 1D code. The current density, which is initially nearly flat about the magnetic axis, begins to increase where the drive term is strong and to decrease at the center until it becomes slightly negative somewhere in the central region (Fig. 3). Note that, as illustrated in the Figure, the current density under these conditions will cross zero off-axis (at a major radius of approximately 2.74) first rather than at the axis (at $R=3.05$ ). 


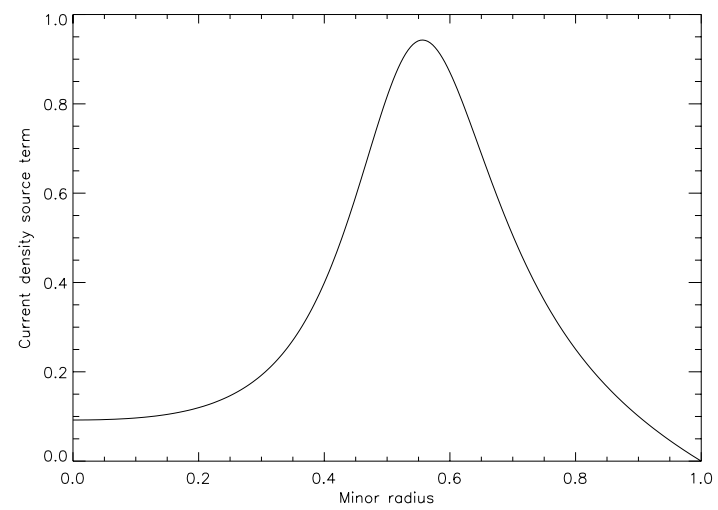

Figure 2: The form of the hollow-current off-axis current drive term.

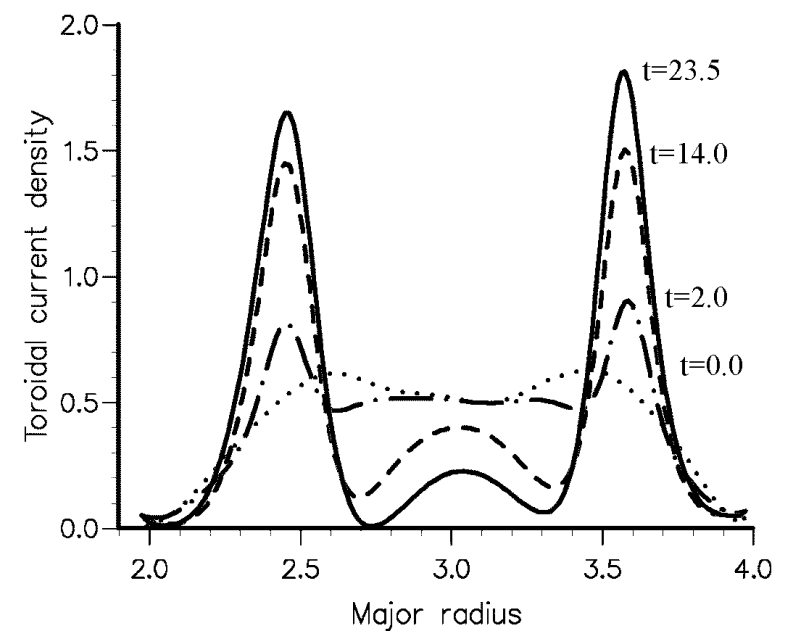

Figure 3: Current density along midplane during initial phase of JET profile evolution. 
This is significant because it means that the $\iota=0$ surface will appear in the plasma not immediately after the current density becomes locally negative, but only after it has become sufficiently negative that the net toroidal current contained within some flux surface is zero.

At the instant the $\iota=0$ surface appears, at finite minor radius, the q-profile is as shown in Fig. 4a. The current inside this surface has not yet reached zero, so there are still good flux surfaces both within and exterior to the surface (Fig. 4b) with a stochastic region in between. One intuitively expects this plasma state to undergo reconnection. The $q=\infty$ region is clearly incapable of supporting the inner set of nested surfaces against the forces that give rise to the Shafranov shift, because the poloidal field vanishes there. This is indeed what we observe (Fig. 5). The central region, which still has finite rotational transform, shifts outward axisymmetrically, slipping past the stochastic lines near the rational surface and reconnecting with the outer region. As a result of the reconnection, the toroidal currents mix, and the outer positive current is rapidly redistributed to the inner region, canceling out its small negative component.

\section{B. Circular cross-section}

As Fig. 5 illustrates, there is a strong poloidal mode number $m=2$ component to the flux contours seen in the simulation using the JET cross-section. We postulated that this was due to the elongation of that equilibrium and switched to a simpler, circular cross-section to eliminate this geometrical effect. As predicted, the behavior of the plasma was similar, but the reconnection now took place as an $m=1$ mode, resembling classic Kadomtsev reconnection, ${ }^{14}$ only axisymmetric (Fig. 6 ).

For sufficiently low viscosity and resistivity, the axisymmetric sawtooth repeats for several cycles. Over the resistivity range $5 \times 10^{-5}<\eta<2 \times 10^{-4}$, the sawtooth period is proportional to $\eta^{-0.55}$, consistent with the expected rate of magnetic reconnection in MHD. As a result of this continual redistribution of current, the central density remains clamped near zero (Fig. 7).

\section{Current Holes in 3D}

In order to investigate the possibility of toroidal dependencies in the sawtooth mode structure, we next extend our circular cross-section study into the third dimension. The qualitative behavior of the plasma in this case is substantially the same as in two dimensions. After the central current density has gone negative and the $\iota=0$ surface has appeared, an $m=1, n=0$ mode sets in and flattens the current at zero. There is also some indication of periodicity (Fig. 8).

However the behavior of the current hole in three dimensions is complicated, as seen in the Figure, by the presence of additional higher $n$ and $m$ modes. For $\eta$ greater than about $5 \times 10^{-5}$, the strongest of these is an $m=4, n=1$ mode localized around the $q=4$ surface, with a linear growth rate of about $0.05 \tau_{A}^{-1}$. At $\eta \approx 3 \times 10^{-5}$, this mode is surpassed in intensity by an $m=3, n=1$ mode at the $q=3$ surface with a growth rate of $0.03 \tau_{A}^{-1}$. Evidently these two modes have different resistivity dependencies.

\section{Summary and Conclusions}

When strong off-axis current drive is applied to the plasma discharges under discussion, the axial current decreases only until it reaches zero or becomes at most slightly negative. At that point, a $q=\infty$ surface appears, leading to an instability in which an axisymmetric 

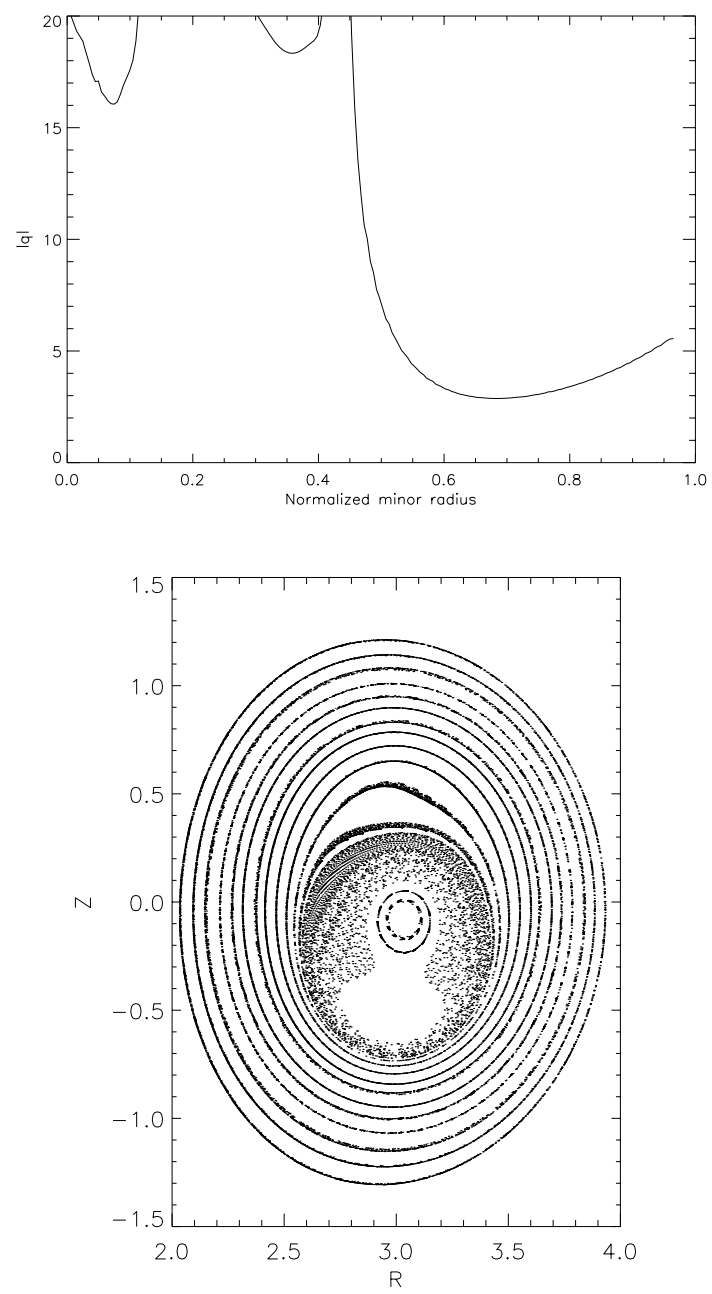

Figure 4: a. Safety factor $(q)$ profile for simulated 2D JET current hole discharge when current density becomes negative. b. Poincaré plot showing locations of flux surfaces at the same instant. 

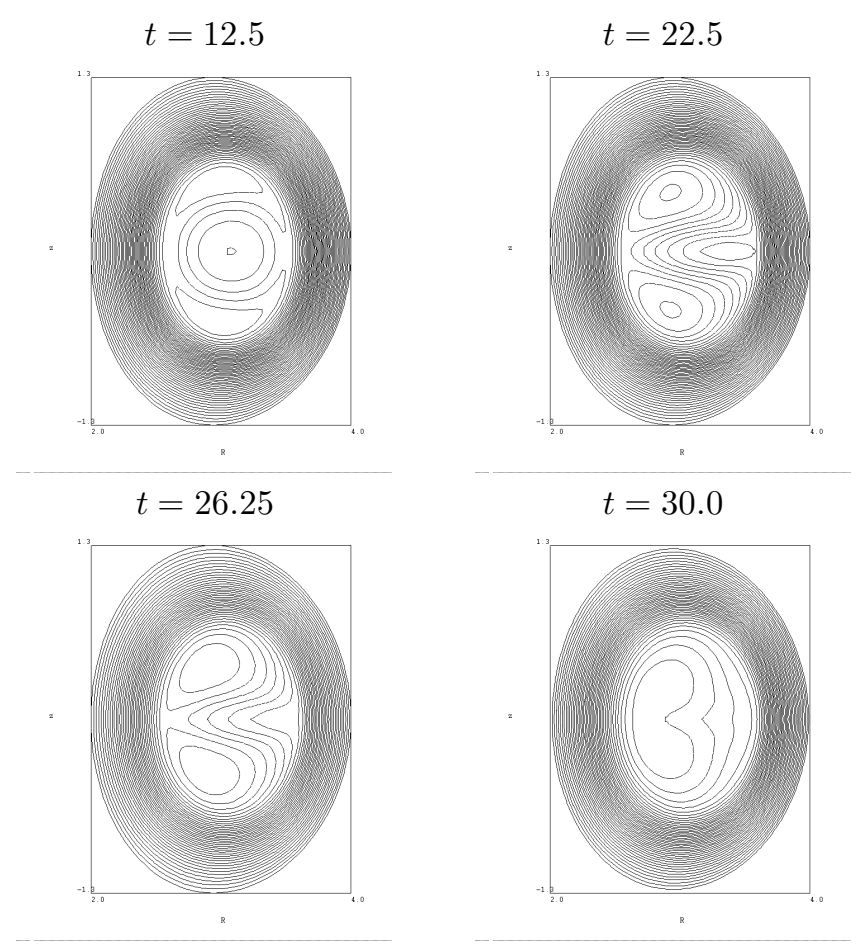

Figure 5: Poloidal flux contours for simulated 2D JET current hole at several times following the appearance of the $\iota=1$ surface, illustrating reconnection. 

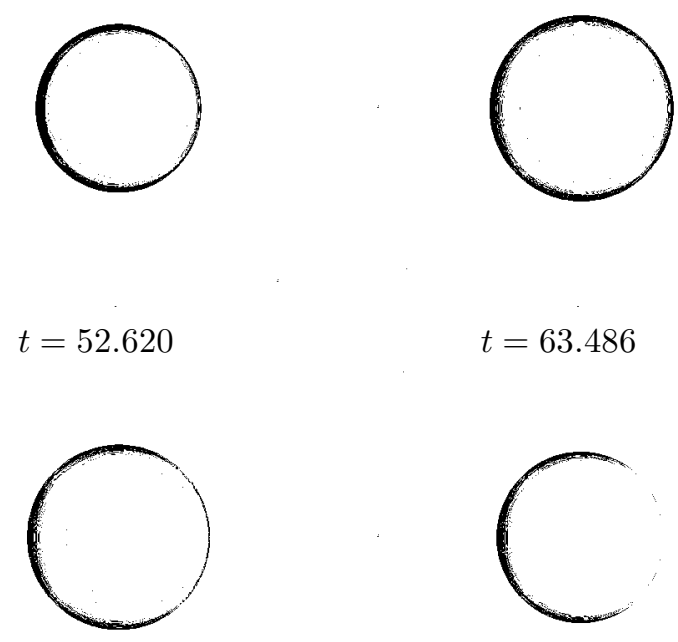

Figure 6: Poloidal flux contours for simulated 2D circular cross-section current hole discharge at several times following the appearance of the $\iota=1$ surface, illustrating 1,0 reconnection. $\eta=\mu=10^{-3}$. Contours are drawn only in the region of nonmonotonic $\psi$ for clarity.

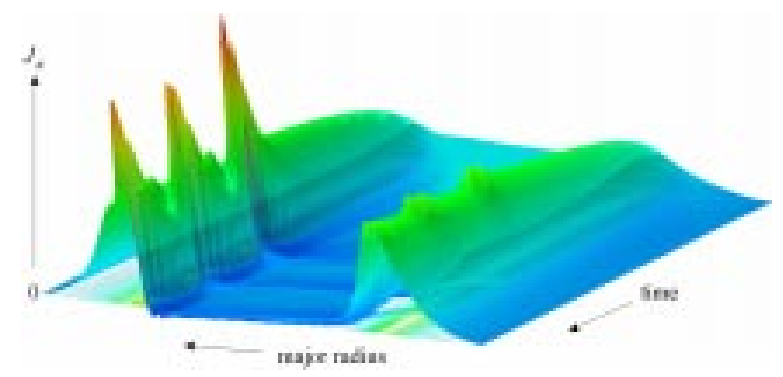

Figure 7: Time history of toroidal current density along midplane during 2D circular crosssection simulation. $\eta=\mu=10^{-4}$. 


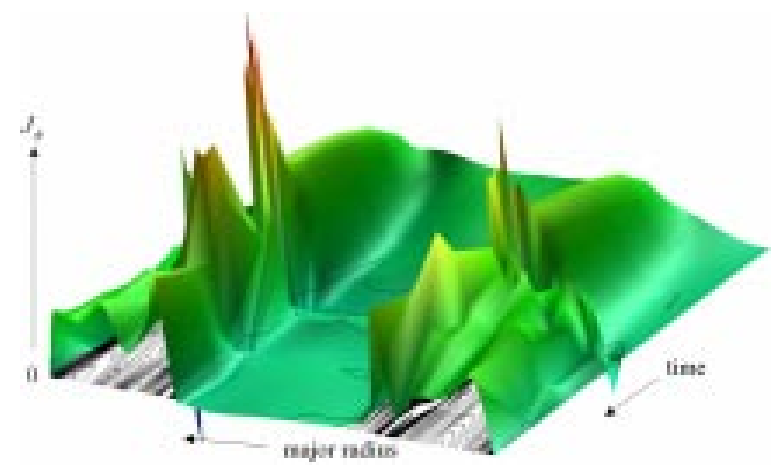

Figure 8: Time history of toroidal current density along midplane at $\phi=0$ during 3D circular cross-section simulation. $\eta=\mu=10^{-4}$.

sawtooth occurs. The sawtooth redistributes the current, increasing its central value until the rational surface again vanishes. If the drive term is maintained, the sawteeth will repeat for several cycles. Over longer times, viscous damping will lead to a steady state with zero core current. On the still longer resistive timescale, the current density will grow again until it matches the drive term and is everywhere positive.

\section{ACKNOWLEDGMENT}

It is a pleasure to acknowledge useful discussions with B. Stratton, L. Zakharov, G. Huysmans, N. Hawkes, and E. Solano. This work was supported by USDOE Contract No. DE-AC020-76CH03073. 


\section{References}

${ }^{1}$ N.C. Hawkes, B.C. Stratton, T. Tala, et al., Phys. Rev. Lett. 87, 115001 (2001).

${ }^{2}$ T. Fujita, T. Oikawa, T. Suzuki, et al., Phys. Rev. Lett. 87, 245001 (2001).

${ }^{3}$ N.C. Hawkes, Y. Andrew, C.D. Challis, et al., Plasma Phys. Control. Fusion 44, 1105 (2002).

${ }^{4}$ C.D. Challis, X. Litaudon, G. Tresset, et al., Plasma Phys. Control. Fusion 44, 1031 (2002).

${ }^{5}$ J.M. Greene, J.L. Johnson, and K.E. Weimer, Phys. Fluids 14, 671 (1971).

${ }^{6}$ M.S. Chu and P.B. Parks, Phys. Plasmas 9, 5036 (2002).

${ }^{7}$ W. Park, E.V. Belova, G.Y. Fu, X.Z. Tang, H.R. Strauss, and L.E. Sugiyama, Phys. Plasmas 61796 (1999).

${ }^{8}$ G.T.A. Huysmans, T.C. Hender, N.C. Hawkes, and X. Litaudon, Phys. Rev. Lett. 87, 245002 (2001).

${ }^{9}$ G.T.A. Huysmans, T.C. Hender, N.C. Hawkes, and X. Litaudon, "MHD stability of 'current hole' plasmas," Joint Varenna-Laussane Workshop on the Theory of Fusion Plasmas (2002).

${ }^{10}$ B.C. Stratton, J.A. Breslau, R.V. Budny, et al., Plasma Phys. Control. Fusion 44, 1127 (2002).

${ }^{11}$ B.C. Stratton, N.C. Hawkes, G.T.A. Huysmans, et al., Proceedings of the 19th IAEA Fusion Energy Conference, Lyon, France (2002).

${ }^{12}$ S. Jardin, N. Pomphrey, and J. DeLucia, J. Comp. Phys. 66481 (1986).

${ }^{13}$ D.W. Ignat, E.J. Valeo, and S.C. Jardin, Nucl. Fusion 34837 (1994).

${ }^{14}$ B.B. Kadomtsev, Sov. J. Plasma Phys. 1389 (1975). 


\section{External Distribution}

Plasma Research Laboratory, Australian National University, Australia

Professor I.R. Jones, Flinders University, Australia

Professor João Canalle, Instituto de Fisica DEQ/IF - UERJ, Brazil

Mr. Gerson O. Ludwig, Instituto Nacional de Pesquisas, Brazil

Dr. P.H. Sakanaka, Instituto Fisica, Brazil

The Librarian, Culham Laboratory, England

Mrs. S.A. Hutchinson, JET Library, England

Professor M.N. Bussac, Ecole Polytechnique, France

Librarian, Max-Planck-Institut für Plasmaphysik, Germany

Jolan Moldvai, Reports Library, MTA KFKI-ATKI, Hungary

Dr. P. Kaw, Institute for Plasma Research, India

Ms. P.J. Pathak, Librarian, Insitute for Plasma Research, India

Ms. Clelia De Palo, Associazione EURATOM-ENEA, Italy

Dr. G. Grosso, Instituto di Fisica del Plasma, Italy

Librarian, Naka Fusion Research Establishment, JAERI, Japan

Library, Plasma Physics Laboratory, Kyoto University, Japan

Research Information Center, National Institute for Fusion Science, Japan

Dr. O. Mitarai, Kyushu Tokai University, Japan

Library, Academia Sinica, Institute of Plasma Physics, People's Republic of China

Shih-Tung Tsai, Institute of Physics, Chinese Academy of Sciences, People's Republic of China

Dr. S. Mirnov, TRINITI, Troitsk, Russian Federation, Russia

Dr. V.S. Strelkov, Kurchatov Institute, Russian Federation, Russia

Professor Peter Lukac, Katedra Fyziky Plazmy MFF UK, Mlynska dolina F-2, Komenskeho Univerzita, SK-842 15 Bratislava, Slovakia

Dr. G.S. Lee, Korea Basic Science Institute, South Korea

Institute for Plasma Research, University of Maryland, USA

Librarian, Fusion Energy Division, Oak Ridge National Laboratory, USA

Librarian, Institute of Fusion Studies, University of Texas, USA

Librarian, Magnetic Fusion Program, Lawrence Livermore National Laboratory, USA

Library, General Atomics, USA

Plasma Physics Group, Fusion Energy Research Program, University of California at San Diego, USA

Plasma Physics Library, Columbia University, USA

Alkesh Punjabi, Center for Fusion Research and Training, Hampton University, USA

Dr. W.M. Stacey, Fusion Research Center, Georgia Institute of Technology, USA

Dr. John Willis, U.S. Department of Energy, Office of Fusion Energy Sciences, USA

Mr. Paul H. Wright, Indianapolis, Indiana, USA 
The Princeton Plasma Physics Laboratory is operated by Princeton University under contract with the U.S. Department of Energy.

\author{
Information Services \\ Princeton Plasma Physics Laboratory \\ P.O. Box 451 \\ Princeton, NJ 08543
}

Phone: 609-243-2750

Fax: 609-243-2751

e-mail: pppl_info@pppl.gov

Internet Address: http://www.pppl.gov 